\title{
Tadek Nadzieja
}

Construction of a smooth Lyapunov function for an asymptotically stable set

Czechoslovak Mathematical Journal, Vol. 40 (1990), No. 2, 195-199

Persistent URL: http://dml.cz/dmlcz/102373

\section{Terms of use:}

(C) Institute of Mathematics AS CR, 1990

Institute of Mathematics of the Czech Academy of Sciences provides access to digitized documents strictly for personal use. Each copy of any part of this document must contain these Terms of use.

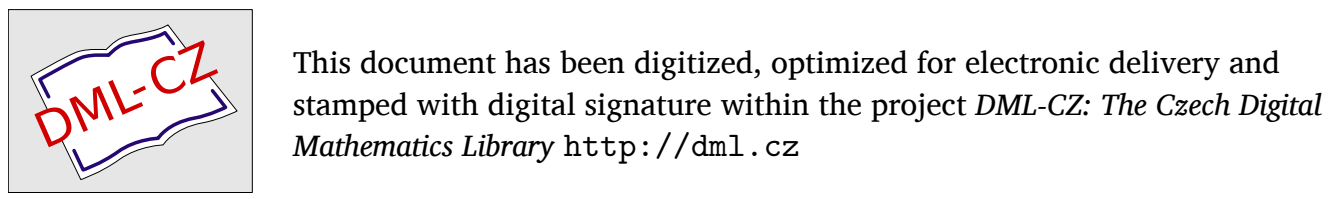




\title{
CONSTRUCTION OF A SMOOTH LYAPUNOV FUNCTION FOR AN ASYMPTOTICALLY STABLE SET
}

\author{
TADEK NADZIEJA, Wrocław \\ (Received June 19, 1986)
}

Let $M$ be a smooth Riemannian manifold and $X$ a complete vector field on $M$ which induces the continuous flow $X(t, \cdot), t \in \mathbb{R}$.

Definition 1. A continuous function $L$ defined on some neighbourhood $U$ of a set $A \subset M$ is a Lyapunov function for $A$ if:

$$
\begin{aligned}
& L(p) \geqq 0 \text { for all } p \in U \text { and } L(p)=0 \text { iff } p \in A, \\
& X L(p)<0 \text { for } p \in U \backslash A \\
& \lim _{n \rightarrow+\infty} L\left(p_{n}\right)=+\infty \text { as } p_{n} \rightarrow \infty
\end{aligned}
$$

Here $p_{n} \rightarrow \infty$ means that for every compact $K \subset U$ there exists $n_{0} \in \mathbb{N}$ such that $p_{n} \notin K$ for $n>n_{0}$.

Let further, for a given $A \subset M$,

$$
D(A)=\{p: \omega-\text { limit set of } p \text { is nonempty and it is contained in } A\} .
$$

Definition 2. A compact, invariant set $A \subset M$ is asymptotically stable if: there exists a neighbourhood $U$ of $A$ such that $U \subset D(A)$, for every neighbourhood $\tilde{W}$ of $A$ there exists a neighbourhood $W$ of $A$ such that $X(t, W) \subset \tilde{W}$ for all $t \geqq 0$.

It is easy to see that in case $A$ is asymptotically stable the set $D(A)$ is open (see [1], Chapter V, Theorem 1,8).

Lyapunov proved that the existence of a Lyapunov function for compact, invariant set implies its asymptotic stability.

It is an interesting question wheter the existence of a smooth Lyapunov function is also a necessary condition for asymptotic stability.

Persidskii [5] gave a partial answer to this problem. He showed that if $x_{0}$ is singular asymptotically stable point of $C^{1}$-vector field, then there exists a $C^{1}$-Lyapunov function for $x_{0}$.

Zubov [7] generalized the above result. He proved that we obtain a $C^{k}$-Lyapunov function if the vector field is $C^{k}$. 
Massera [3], [4] showed the existence of Lyapunov function for asymptotically stable point which has partial derivatives of any order assuming that the vector field satisfies the Lipschitz condition.

Kurzweil [2] proved a similar theorem under weaker assumptions about vector field.

The problem has been completely solved by Wilson [6]. Namely, using the new technique of approximation of a continuous function by a smooth one he proved the following

Theorem (cf [6], Theorems 3.1 and 3.2). If $A$ is an asymptotically stable set, then there exists a $C^{\infty}$-Lyapunov function for $A$ defined on $D(A)$.

Unfortunately, there are some gaps in Wilson's proof, for example, it is not obvious that for the function $U$ defined in the proof of Theorem 3.1 $X U$ exists.

Our aim is to give a new proof of the Theorem. The method used in the paper is based on the following main result of [6].

Lemma (cf [6], Theorem 2.5). If $f$ is a continuous real-valued function on an open set $U \subset M, X$ is a nonsingular vector field on $U$, and if $X f$ is defined and continuous, then for every positive continuous function $\varepsilon: U \rightarrow \mathbb{R}$ there exists a $C^{\infty}$-function $g: U \rightarrow \mathbb{R}$ such that

$$
|f(x)-g(x)|<\varepsilon(x) \text { and } X g(x)<X f(x)+\varepsilon(x)
$$

for all $x \in U$.

In the sequel the following Proposition will prove useful.

Proposition. Let $K$ be a compact subset of $D(A)$. Then for every neighbourhood $\widetilde{W}$ of $A$ there exist a neighbourhood $W_{K}$ of $K$ and a number $T>0$ such that $X\left(t, W_{K}\right) \subset$ $\subset \tilde{W}$ for all $t \geqq T$.

Proof. It follows from (2b) that there is a neighbourhood $W$ od $A$ such that $X(t, W) \subset \tilde{W}$ for all $t \geqq 0$.

Suppose now that a point $p \in D(A)$. Then there exists $T_{p}>0$ such that $X\left(T_{p}, p\right) \in W$ and so, by the continuity of the flow, $X\left(T_{p}, W_{p}\right) \subset W$ for some neighbourhood $W_{p}$ of $p$. Hence $X\left(t, W_{p}\right) \subset \widetilde{W}$ for all $t \geqq T_{p}$.

Thus for any $p \in K$ there exist a neighbourhood $W_{p}$ of $p$ and $T_{p}>0$ such that $X\left(t, W_{p}\right) \subset \tilde{W}$ for all $t \geqq T_{p}$. Now the Proposition follows from the compactness of $K$.

Now we are ready to prove two remarks which we need for the proof of Theorem.

As an easy consequence of Proposition we will get the following

Remark 1. Suppose that $\left\{p_{n}\right\} \subset D(A), \operatorname{cl}\left\{p_{n}\right\} \cap A=\emptyset$, and $t_{n} \leqq T_{0}$ for some $T_{0} \in \mathbb{R}$. If the sequence $\left\{X\left(t_{n}, p_{n}\right)\right\}$ is contained in a compact subset of $D(A)$ then the sequence $\left\{t_{n}\right\}$ is bounded.

Proof. Let $\tilde{W}$ be a neighbourhood of $A$ for which $\widetilde{W} \cap \operatorname{cl}\left\{p_{n}\right\}=\emptyset$. It follows from Proposition that there exists $T>0$ such that $X\left(t+t_{n}, p_{n}\right) \in \widetilde{W}$ for all $t \geqq T$. Thus $t_{n}>-T$. 
Remark 2. Suppose that $\left\{p_{n}\right\} \subset D(A)$ and $t_{n} \leqq T_{0}$ for some $T_{0} \in \mathbb{R}$. If $p_{n} \rightarrow \infty$ then $X\left(t_{n}, p_{n}\right) \rightarrow \infty$ too.

Proof. Suppose it is not. Thus there exists a sequence $\left\{p_{n_{k}}\right\}$ such that $p_{n_{k}} \rightarrow \infty$ and $\operatorname{cl}\left\{X\left(t_{n_{k}}, p_{n_{k}}\right)\right\}$ is a compact subset of $D(A)$ while, by Remark 1 , the sequence $\left\{-t_{n_{k}}\right\}$ is bounded. But this contradicts the continuity of the flow.

Proof of Theorem. We equip the set $D(A)$ with a complete Riemannian metric $\varrho$. In the case the closed, bounded sets are compact, and so ( $A$ is compact) a sequence $\left\{p_{n}\right\}, p_{n} \in D(A)$, is contained in a compact subset of $D(A)$ iff the sequence $\operatorname{dist}\left(p_{n}, A\right)$ is bounded.

Next we consider the function $\varphi: D(A) \rightarrow \mathbb{R}^{+}$defined by

Obviously,

$$
\varphi(p)=\inf \left\{\operatorname{dist}_{\varrho}(X(t, p), A): t \leqq 0\right\} .
$$

$$
\varphi(X(t, p)) \leqq \varphi(p) \text { for all } t \geqq 0
$$

and

$$
\varphi(p) \leqq \operatorname{dist}_{\varrho}(p, A) .
$$

It follows from Remark 1 that if $p \in D(A) \backslash A$ then $\lim _{t \rightarrow-\infty} \operatorname{dist}_{\varrho}(X(t, p), A)=+\infty$. Therefore there exists a nonpositive function $\tilde{t}$ on $D(A)$ such that

$$
\varphi(p)=\operatorname{dist}_{\varrho}(X(\tilde{t}(p), p), A) .
$$

Hence, in particular, we get

$$
\varphi(p)=0 \text { iff } p \in A \text {. }
$$

We claim that $\varphi$ is continuous on $D(A)$. Obviously $\varphi$ is continuous at points $p \in A$. Suppose now that $p \in D(A) \backslash A$ and a sequence $\left\{p_{n}\right\}$ goes to $p$. From the continuity of the flow we get

$$
\varphi(p)=\operatorname{dist}_{\varrho}(X(\tilde{t}(p), p), A) \geqq \limsup _{n \rightarrow+\infty} \varphi\left(p_{n}\right) .
$$

On the other hand, by the last inequality the sequence $\varphi\left(p_{n}\right)=\operatorname{dist}_{\varrho}\left(X\left(\tilde{t}\left(p_{n}\right), p_{n}\right), A\right)$ is bounded, and so, in view of Remark 1 , the sequence $\left\{\tilde{t}\left(p_{n}\right)\right\}$ is bounded. This and the continuity of the flow imply that

$$
\varphi(p) \leqq \liminf _{n \rightarrow+\infty} \varphi\left(p_{n}\right) .
$$

Thus $\varphi\left(p_{n}\right) \rightarrow \varphi(p)$.

Suppose next that $\left\{p_{n}\right\} \subset D(A)$ and $p_{n} \rightarrow \infty$. It follows from Remark 2 that $\operatorname{dist}_{o}\left(X\left(\tilde{t}\left(p_{n}\right), p_{n}\right), A\right) \rightarrow+\infty$. Thus we get

$$
\text { if } p_{n} \rightarrow \infty \text { then } \varphi\left(p_{n}\right) \rightarrow+\infty \text {. }
$$

Let now $\varphi$ be a nonnegative continuous function defined on $D(A)$ with the properties (3abcd). Define the function $\Phi: D(A) \rightarrow \mathbb{R}^{+}$by

$$
\Phi(p)=\int_{0}^{+\infty} \varphi(X(\tau, p)) \exp (-\tau) \mathrm{d} \tau .
$$


Obviously $\Phi(p)=0$ iff $p \in A$.

We claim that $\Phi$ is continuous on $D(A)$.

In fact, suppose $p, \tilde{p} \in D(A)$. For every $T>0$ we have

$$
\begin{aligned}
& \Phi(p)-\Phi(\tilde{p})=\int_{0}^{+\infty}[\varphi(X(\tau, p))-\varphi(X(\tau, \tilde{p}))] \exp (-\tau) \mathrm{d} \tau= \\
& =\int_{0}^{T}[\varphi(X(\tau, p)-\varphi(X(\tau, \tilde{p}))] \exp (-\tau) \mathrm{d} \tau+ \\
& +\int_{T}^{+\infty}\left[\varphi(X(\tau, p)-\varphi(X(\tau, \tilde{p}))] \exp (-\tau) \mathrm{d} \tau=: I_{1}+I_{2} .\right.
\end{aligned}
$$

It is easy to see that for sufficiently large $T, I_{2}$ is small for $\tilde{p}$ from some neighbourhood of $p$. If $T$ is fixed then $I_{1}$ is small for $\tilde{p}$ from some neighbourhood of $p$. Thus $\Phi$ is continuous at the point $p$.

We now calculate $X \Phi(p)$ :

$$
\begin{aligned}
& \frac{1}{t}[\Phi(X(t, p))-\Phi(p)]= \\
& =\frac{1}{t}\left[(\exp t-1) \int_{t}^{+\infty} \varphi(X(\tau, p)) \exp (-\tau) \mathrm{d} \tau+\int_{0}^{t} \varphi(X(\tau, p)) \exp (-\tau) \mathrm{d} \tau\right] .
\end{aligned}
$$

Letting $t \rightarrow 0$, we get

$$
X \Phi(p)=\Phi(p)-\varphi(p) .
$$

We claim that $\Phi(p)<\varphi(p)$ for $p \in D(A) \backslash A$.

In fact, by (3a)

$$
\Phi(p)=\int_{0}^{+\infty} \varphi(X(\tau, p)) \exp (-\tau) \mathrm{d} \tau \leqq \int_{0}^{+\infty} \varphi(p) \exp (-\tau) \mathrm{d} \tau=\varphi(p) .
$$

Suppose $\Phi(p)=\varphi(p)$. Hence $\varphi(X(\tau, p))=\varphi(p)$ for all $\tau \geqq 0$. Thus $\varphi(p)=$ $=\lim _{\tau \rightarrow+\infty} \varphi(X(\tau, p))=0$ and, consequently, $p \in A$.

Thus we have shown that $X \Phi(p)<0$ for $p \in D(A) \backslash A$.

Next we will prove that $\Phi$ satisfies the condition (1c).

Suppose that $p_{n} \rightarrow \infty$. It follows from (3a) that $\varphi\left(X\left(\tau, p_{n}\right)\right)$ are nonincreasing functions of $\tau$. Thus we have

$$
\begin{aligned}
\Phi\left(p_{n}\right) & =\int_{0}^{+\infty} \varphi\left(X\left(\tau, p_{n}\right)\right) \exp (-\tau) \mathrm{d} \tau \geqq \int_{0}^{1} \varphi\left(X\left(\tau, p_{n}\right)\right) \exp (-\tau) \mathrm{d} \tau \geqq \\
& \geqq \varphi\left(X\left(1, p_{n}\right)\right) \int_{0}^{1} \exp (-\tau) \mathrm{d} \tau .
\end{aligned}
$$

At the same time (see Remark 2), $X\left(1, p_{n}\right) \rightarrow \infty$. Hence, by (3d), $\Phi\left(p_{n}\right) \rightarrow+\infty$.

We have shown that $\Phi$ is a Lyapunov function for $A$. Moreover, by (3b) and (4), $\Phi$ satisfies the condition

$$
\Phi(p) \leqq \operatorname{dist}_{e}(p, A) .
$$

Finally, suppose that we have a Lyapunov function $\Phi$ for $A$ defined on $D(A)$ and satisfying the condition (5). It follows from Lemma that there exists a $C^{\infty}$-function $\tilde{L}: D(A) \backslash A \rightarrow \mathbb{B}^{+}$such that

$$
|\tilde{L}(p)-\Phi(p)|<\frac{1}{2} \Phi(p) \text { and } X \tilde{L}(p)<\frac{1}{2} X \Phi(p)
$$


(we put in Lemma $\left.\varepsilon(p)=\min \left(\frac{1}{2} \Phi(p), \frac{1}{2} X \Phi(p)\right)\right)$. Moreover, by (5),

$$
\tilde{L}(p) \leqq C \operatorname{dist}_{e}(p, A) \text { for some constant } C \text {. }
$$

Now we define the function $L$ on $D(A)$ by

$$
L(p)=\left\{\begin{array}{cll}
{[\exp (-1 / \tilde{L}(p))] \tilde{L}(p)} & \text { for } & p \in D(A) \backslash A, \\
0 & \text { for } & p \in A .
\end{array}\right.
$$

Obviously $L$ is a Lyapunov function for $A$ which is $C^{\infty}$ on $D(A) \backslash A$. We claim that at points $p \in A L$ is $C^{\infty}$ too.

In fact, the inequality (6) implies that

$$
L(p)=o\left(\left(\operatorname{dist}_{\varrho}(p, A)\right)^{k}\right) \text { for all } k>0,
$$

where $o\left(h^{k}\right)$ denotes a function for which

$$
\lim _{h \rightarrow 0} \frac{o\left(h^{k}\right)}{h^{k}}=0 \text {. }
$$

Hence $L$ has all derivatives 0 at every point $p \in A$.

This finishes the proof of our theorem.

Acknowledgment. I am indebted to dr B. Mincer for helpful discussions during preparation of this paper.

\section{References}

[1] N. P. Bhatia, G. P. Szegö: Stability Theory of Dynamical Systems, Springer-Verlag, BerlinHeidelberg-New York 1970.

[2] J. Kurzweil: On the inversion of Lyapunov's second theorem on stability of motion, Czechoslovak Math. J. 81 (1956), 217-259, 455-473 (in Russian; Summary in English, ibidem, 474-484). English transl.: Amer. Math. Soc. Transl., Ser. II, 24 (1963), 19-77.

[3] J. L. Massera: Contributions to stability theory, Ann. of Math. 64 (1956), 182-206; Erratum, Ann. of Math. 68 (1958), 202.

[4] J. L. Massera: Converse theorems of Lyapunov's second method, Symp. Intern. Ecuac. Dif. Ordin., Mexixo 1961 (1962), 158-163.

[5] K. P. Persidskii: On a theorem of Lyapunov, Dokl. Akad. Nauk SSSR 14 (1937), 541-544 (in Russian).

[6] F. W. Wilson: Smoothing derivatives of functions and applications, Trans. Am. Math. Soc. 139 (1969), 413-428.

[7] V. I. Zubov: Methods of A. M. Lyapunov and their Application, Leningrad 1957 (in Russian). English transl.: Groningen 1964.

Author's address: Mathematical Institute University of Wrockaw, pl. Grunwaldzki 2/4, 50-384 Wrockaw, Poland. 